\title{
The benefit of platelet-rich plasma injection over institution- based physical therapy program in adhesive capsulitis patients with diabetes mellitus: prospective observational cohort study
}

\author{
Apurba Barman ${ }^{1}$, Somnath Mukherjee ${ }^{2}$, Mithilesh K Sinha ${ }^{3}$, Jagannatha Sahoo ${ }^{1}$, Amrutha Viswanath ${ }^{1}$ \\ ${ }^{1}$ Department of Physical Medicine and Rehabilitation, All India Institute of Medical Sciences, Bhubaneswar, India \\ ${ }^{2}$ Department of Transfusion Medicine, All India Institute of Medical Sciences, Bhubaneswar, India \\ ${ }^{3}$ Department of General Surgery, All India Institute of Medical Sciences, Bhubaneswar, India
}

\begin{abstract}
Background: The objective of this study was to compare the efficacy of platelet-rich plasma (PRP) injection with an institution-based physical therapy (PT) program for adhesive capsulitis (AC) of the shoulder in patients with diabetes mellitus (DM).

Methods: A total of seventy diabetic patients with AC of the shoulder for $<6$ months were assigned to two groups: PRP group and PT group. In the PRP group, 35 patients were administered a single shot of PRP (4 mL) into the glenohumeral joint. In the PT group, 35 patients were given institution-based PT that included 10 30-minute sessions of planned PT over a 2-week period. After the interventions, all patients were prospectively followed for 12 weeks. Intensity of shoulder pain, function, and range of motion were assessed at baseline and then at 3,6 , and 12 weeks.

Results: Thirty-three patients in the PRP group and 32 in the PT group completed the 12-week study. At 12 weeks, patients who received PRP injections showed greater improvement in shoulder pain $(\mathrm{p}<0.001)$ than those recruited to the PT group. In the range of motion and shoulder function activities, patients in the PRP group showed significant improvement compared with the institution-based PT group $(\mathrm{p}<0.001)$. No significant complications were reported from any groups.

Conclusions: In a diabetic population, PRP injections significantly improved shoulder pain and function compared with an institution-based PT program for shoulder AC. Additionally, it is a safe and well-tolerated method for AC management for diabetic patients.
\end{abstract}

Keywords: Shoulder; Diabetes mellitus; Platelet-rich plasma; Physical therapy; Ultrasound therapy

\section{INTRODUCTION}

Adhesive capsulitis (AC), one of the common painful musculoskeletal conditions, presents with impaired shoulder function and movement restrictions of the glenohumeral joint $[1,2]$. The incidence of primary AC is approximately $2 \%-5 \%$ in the general population, and can be as high as $20 \%$ among diabetic people [3]. Diabetes mellitus (DM) has been reported as the most common cause of secondary AC [3]. Patients with DM and AC have been reported as having worse functional outcomes, including disabil-

Received: June 6, $2021 \quad$ Revised: July 1, $2021 \quad$ Accepted: July 8, 2021

Correspondence to: Apurba Barman

Department of Physical Medicine and Rehabilitation, All India Institute of Medical Sciences, Bhubaneswar, Odisha 751019, India

Tel: +91-943-8884211, Fax: +91-674-2470331, E-mail: apurvaa23@gmail.com, ORCID: https://orcid.org/0000-0002-8990-1731

\section{Financial support: None.}

Conflict of interest: None.

Copyright@ 2021 Korean Shoulder and Elbow Society.

This is an Open Access article distributed under the terms of the Creative Commons Attribution Non-Commercial License (http://creativecommons.org/licenses/by-nc/4.0/) which permits unrestricted non-commercial use, distribution, and reproduction in any medium, provided the original work is properly cited. 
ity, compared with patients without DM [4].

Therapeutic exercises, particularly joint mobilization and stretching, are the mainstay of conservative AC treatment [5]. However, these therapeutic exercises may aggravate pre-existing shoulder pain during mobilization. Therefore, these exercises are frequently advised along with various pain-relieving agents, such as oral/injectable medications or pain-relieving physical modalities.

Several injections, including intra-articular corticosteroid (CS) injection [2], sodium hyaluronate injection $[3,6]$, and hydraulic distension (hydrodilatation) [7], have been advocated to reduce shoulder pain. However, patients with DM often express concern about the side effects from these injections, which include hyperglycemia, subcutaneous tissue atrophy, and tendon rupture) of CS injection [8]. Furthermore, sodium hyaluronate injection is expensive. Therefore, many patients with DM and AC prefer to receive ultrasound therapy (UST) or transcutaneous electrical nerve stimulation (TENS), or to continue to undergo various combinations of physical modalities, especially from physical therapy (PT) centers or hospitals, for short-term and long-term care.

A recent new innovative treatment method, platelet-rich plasma (PRP) injection, has received attention for treating musculoskeletal conditions because of its safety and effectiveness across many populations [9-15]. Additionally, many studies have shown its effectiveness for managing shoulder pathologies [15-18]. However, its effectiveness compared with an institution-based PT program has not been evaluated yet, especially for patients with $\mathrm{AC}$ and DM.

The objective of this study was to assess the effectiveness of PRP injections with institution-based PT programs for treating shoulder AC among diabetic patients. We hypothesized that a single shot of intra-articular PRP injection would be superior to an institution-based PT program.

\section{METHODS}

The study was approved by approved by the Institutional Review Board of All India Institute of Medical Sciences, Bhubaneswar, India. Written informed consents were obtained from all participants.

\section{Participants}

Patients with DM and shoulder pain who visited the Physical Medicine and Rehabilitation Out-Patient Department at All India Institute of Medical Sciences between March 2018 to March 2020 were recruited. DM was diagnosed based on either fasting- and 2-hour postprandial-plasma glucose value (75-g oral glucose tolerance test) or glycosylated haemoglobin (HbA1c) values [19]. Patients who were on hypoglycemic agents were considered diabetic. Patients $\geq 18$ years old presenting with shoulder pain and stiffness for less than six months and with $>25 \%$ restriction in passive movement of the shoulder joint in at least two directions (out of shoulder-abduction, -flexion, -internal and -external rotations) compared with the opposite shoulder were included in this study. The inclusion criteria for AC were set according to previous reports $[15,20]$.

Patients with secondary AC were excluded if they had (1) low hemoglobin $\leq 9.9 \mathrm{gm} / \mathrm{dL}$ (moderate to severe anemia), (2) inflammatory arthritis, (3) bony deformities or pre-existing musculoskeletal disorders of the shoulder joint, (4) weakness of the shoulder girdle muscles associated with neurological deficits, (5) cognitive deficits that made them unable to correctly adhere to exercise programs, (6) received an injection for shoulder pain, or (7) undergone any surgeries or invasive procedures in the affected shoulder.

\section{Study Design}

This prospective observational cohort study was conducted at a tertiary care teaching hospital in India. Institutional ethics committee permission was obtained before starting the project. After obtaining written informed consent, patients with DM and AC who fulfilled the inclusion criteria were included in the study. Patients were given the option to undergo either PRP injection or an institution-based PT program. After an initial assessment, patients who were willing to receive a PRP injection were included in the PRP group $(n=35)$. Patients who were not willing to receive the injection (immediately after assessment) but still wanted to participate in the study were included in the PT group $(n=35)$. Patients in the PRP group were given a single intra-articular injection of PRP. Patients in the PT group were administered ten sessions of UST and TENS for 30 minutes/day over a 2 -week period. Based on previously published reports [16], ten sessions of PT over a 2-week period is considered the optimal duration for AC treatment.

PRP solution was prepared using the Eppendorf AG Centrifuge 5702 (Eppendorf Hamburg, Germany; Platelet Separation System). Using an 18-g needle, $25 \mathrm{~mL}$ of venous blood was obtained from each patient, and $24 \mathrm{~mL}$ of venous blood was transferred into two disposable bio-kit tubes (12 mL each) with 1.5 $\mathrm{mL}$ of ACD-A anticoagulant. The remaining 1-mL blood sample was sent for platelet count. Bio-kit tubes were then centrifuged for 14 minutes at $1,800 \mathrm{rpm}$. A total of $5 \mathrm{~mL}$ of PRP was obtained from the tubes. Out of $5 \mathrm{~mL}$ of PRP, $1 \mathrm{~mL}$ was sent for total plate- 
let count, and $4 \mathrm{~mL}$ was held for injection into the target joint. The entire preparation procedure was conducted inside a classIIA biosafety cabinet and under the supervision of a transfusion medicine physician in the clinical laboratory of the Department of Transfusion Medicine. Mean platelet \& leukocyte count (in PRP solution) yields were $694 \times 10^{3} / \mu \mathrm{L}$ and $0.3 \times 10^{3} / \mu \mathrm{L}$ (range, $0.1-1.5 \times 10^{3} / \mu \mathrm{L}$ ), respectively. Both platelet count times (from whole blood and PRP) were conducted using the same automated cell counter (Sysmex XP-100; Sysmex, Kobe, Japan). The injection was then administered into the target shoulder joint within 30 minutes of PRP preparation.

\section{Interventions}

PRP injections $(4 \mathrm{~mL})$ were given intra-articularly through a posterior approach under proper aseptic conditions. One experienced physician performed all injections under ultrasound guidance. An ultrasound machine, SonoSite M-Turbo, and a linear array transducer $(13-6 \mathrm{MHz})$ were used during this intervention. The institution-based PT program consisted of TENS and UST, followed by passive joint mobilization of the affected shoulder. A total of 10 sessions, 30 minutes/day, over 2 weeks (5 days/wk) were administered at our institution. The same physical therapist applied TENS and UST and performed mobilization exercises for all the patients. TENS was applied for 20 minutes and UST for 7 minutes in each session. TENS and UST were administered from the same Intelect Transport Combination Therapy Unit (Chattanooga Chattanooga Intelect Transport, USA) on the anterior and posterior sides of the target shoulder joint. TENS was applied at a $100-\mathrm{Hz}$ frequency and a $15-\mathrm{mA}$ amplitude for 100 milliseconds. UST was given using a transducer head $5 \mathrm{~cm}^{2}$ in area, at a $1-\mathrm{MHz}$ frequency and $1.5-\mathrm{W} / \mathrm{cm}^{2}$ intensity.

The home exercise program, advised to both groups, was demonstrated by the same physical therapist. Each participant was instructed to complete the 20-minute home exercise program daily. The home exercise program included Codman exercises, stretching, and isometric strengthening exercises for the primary shoulder muscles. Non-steroidal anti-inflammatory drugs were not provided to any patient. However, patients were permitted to consume oral acetaminophen $(1 \mathrm{~g})$ up to a maximum of $2 \mathrm{~g} /$ day and to apply a hot water bag to the affected shoulder joint when experiencing severe pain or discomfort. After injection, patients were instructed to rest the intervention arm for 2 days from any overhead activities and rotational movements of the shoulder joint. After 2 days, patients were advised to start home exercises as demonstrated. Participants were called at frequent intervals to encourage home exercises and were advised not to take any pain-relieving medication or any physical agents (pain-relieving modalities).

\section{Outcome Assessment}

The visual analog scale (VAS; $100 \mathrm{~mm}$ ) pain score [16] was used as the primary outcome measure ( 0 , no pain; 100 , worst pain possible). The shoulder pain and disability index (SPADI) and range of motion (ROM) score (both active- and passive-ROM) were used for secondary outcomes [16]. Baseline and outcome assessments at all follow-up visits were performed by one physician who was not associated with the study. Follow-up visits were done at 3 weeks, 6 weeks, and 12 weeks. During their evaluation, all patients were asked to complete the SPADI questionnaire. Additionally, active and passive ROMs (shoulder-flexion, extension, abduction, internal and external rotation) were measured by goniometry.

The SPADI questionnaire [7] consisted of two divisions (pain and disability), with five questions in one division (pain) and eight questions in the second division (disability), for a total of 13 questions (total SPADI). The two division scores were expressed as percentages, and their means were averaged to get a total SPADI score that ranged from $0-100$.

\section{Statistical Analysis}

A sample size calculation was done considering VAS pain score as the primary outcome. To achieve $80 \%$ study power, a 10 -point difference in VAS improvement between the two groups would need to be detected (pooled standard deviation, 14; two-sided t-test $\alpha, 0.05)$. Thirty participants in each group were required to achieve this target.

Categorical variables are presented as percentages or proportions. Continuous variables were presented as the mean (standard deviation). Statistical analyses included intention-to-treat (ITT) analysis [21], and repeated measure analysis of variance and the post-hoc Bonferroni test were used to identify differences in parameter changes at different time points and compare the parameter changes from baseline and 2nd, 3rd, and 4th visits among the two groups. A p-value $<0.05$ was considered statistically significant. Statistical analyses were performed using IBM SPSS ver. 22.0 (IBM Corp., Armonk, NY, USA).

\section{RESULTS}

A total of 70 patients were recruited for this study, 35 patients in each group. Thirty-three patients in the PRP group and 32 in the PT group finished the 12-week study period. Two patients from the PRP group and three from the PT group did not complete all follow-up. A schematic diagram of the study is present- 
ed in Fig. 1. Study participants' demographic and clinical characteristics are reported in Table 1. Figs. 2 and 3 show the distribution of patients' diabetic medications in the PRP and PT groups, respectively.

There were no disparities in baseline characteristics-age, body-mass index, shoulder pain duration, DM duration, blood sugar level, VAS pain score, shoulder ROM, and SPADI score (total)-between the two groups (Table 1). The right shoulder was predominantly affected in both groups.

Both groups showed a decrease in VAS pain scores at every follow-up visit (Table 2). The differences in mean VAS pain scores within the groups from 1st (baseline visit) to 2 nd visit (3 weeks), 1st to 3 rd visit (6 weeks), and 1st to 4 th visit (12 weeks) were statistically significant (Table 3 ). In inter-group comparisons, the improvements in mean VAS pain scores between 1st to 2nd visit, 1 st to $3 \mathrm{rd}$ visit, and 1 st to 4 th visit were greater in the PRP group (Table 3). At the end of 12 weeks, significant differences were observed between the change of the mean VAS pain scores between the PRP and PT groups ( $\mathrm{p} \leq 0.001)$ (Table 4).

With progression of the study, active and passive ROM improved in each group (Table 2). The differences in changes of ROM (flexion, extension, abduction, internal rotation, and external rotation) within the groups from 1 st to $2 \mathrm{nd}$, 1 st to $3 \mathrm{rd}$, and

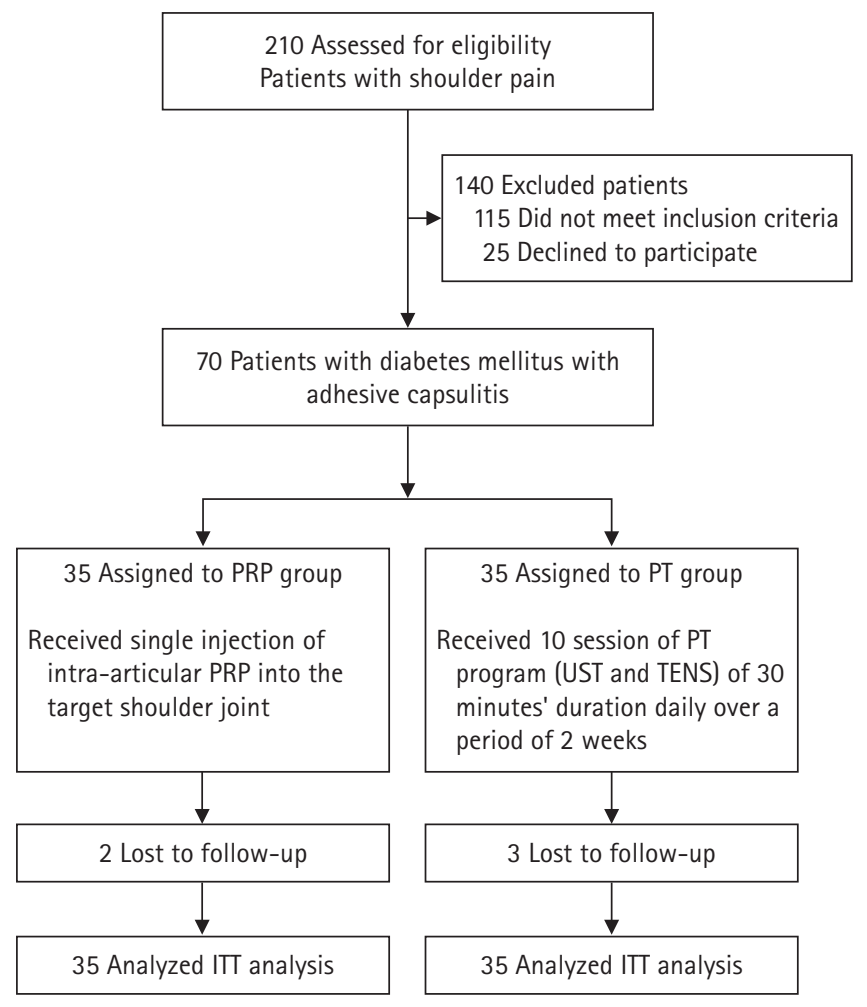

Fig. 1. Flowchart of patient enrollment. PRP: platelet-rich plasma, PT: physical therapy, UST: ultrasound therapy, TENS: transcutaneous electrical nerve stimulation, ITT: intention-to-treat.

Table 1. Baseline demographics and clinical characteristics

\begin{tabular}{|c|c|c|c|}
\hline Parameter & PRP group $(n=35)^{*}$ & PT group $(n=35)^{*}$ & p-value ${ }^{\dagger}$ \\
\hline Total number of patients recruited & 35 & 35 & \\
\hline Patients completed all follow-up & 30 & 29 & \\
\hline Male:female & $14: 21$ & $15: 20$ & 0.90 \\
\hline Age $(y r)$ & $48.8 \pm 5.9$ & $49.6 \pm 5.7$ & 0.55 \\
\hline Body mass index $\left(\mathrm{kg} / \mathrm{m}^{2}\right)$ & $24.9 \pm 2.9$ & $25.4 \pm 3.4$ & 0.57 \\
\hline Duration of disease (mo) & $4.1 \pm 0.9$ & $4.0 \pm 1.0$ & 0.28 \\
\hline VAS & $73.14 \pm 8.5$ & $74.4 \pm 7.5$ & 0.51 \\
\hline \multicolumn{4}{|l|}{ Active $\operatorname{ROM}\left({ }^{\circ}\right)$} \\
\hline Flexion & $80.6 \pm 12.0$ & $78.4 \pm 11.7$ & 0.43 \\
\hline Extension & $22.3 \pm 6.2$ & $21.4 \pm 2.9$ & 0.47 \\
\hline Abduction & $68.0 \pm 8.3$ & $64.8 \pm 9.1$ & 0.12 \\
\hline Internal rotation & $16.8 \pm 6.2$ & $18.0 \pm 5.3$ & 0.39 \\
\hline External rotation & $17.8 \pm 3.8$ & $19.1 \pm 5.4$ & 0.21 \\
\hline \multicolumn{4}{|l|}{ Passive $\mathrm{ROM}\left(^{\circ}\right)$} \\
\hline Flexion & $97.9 \pm 13.5$ & $95.0 \pm 10.6$ & 0.33 \\
\hline Extension & $29.0 \pm 4.5$ & $28.4 \pm 3.7$ & 0.52 \\
\hline Abduction & $88.2 \pm 10.9$ & $86.1 \pm 9.8$ & 0.40 \\
\hline Internal rotation & $25.4 \pm 5.4$ & $26.6 \pm 5.4$ & 0.34 \\
\hline External rotation & $25.0 \pm 6.2$ & $26.2 \pm 4.6$ & 0.35 \\
\hline SPADI & $71.1 \pm 12.0$ & $69.3 \pm 10.3$ & 0.51 \\
\hline
\end{tabular}

Values are presented as mean \pm standard deviation. The p-values correspond to the mean difference between two groups.

PRP: platelet-rich plasma, PT: physical therapy, VAS: visual analog scale, ROM: range of motion, SPADI: shoulder pain and disability index.

${ }^{*}$ Intention-to-treat analysis; ${ }^{\dagger}$ Unpaired t-test/Fischer's exact test for two group comparisons $(\mathrm{p}<0.05)$. 


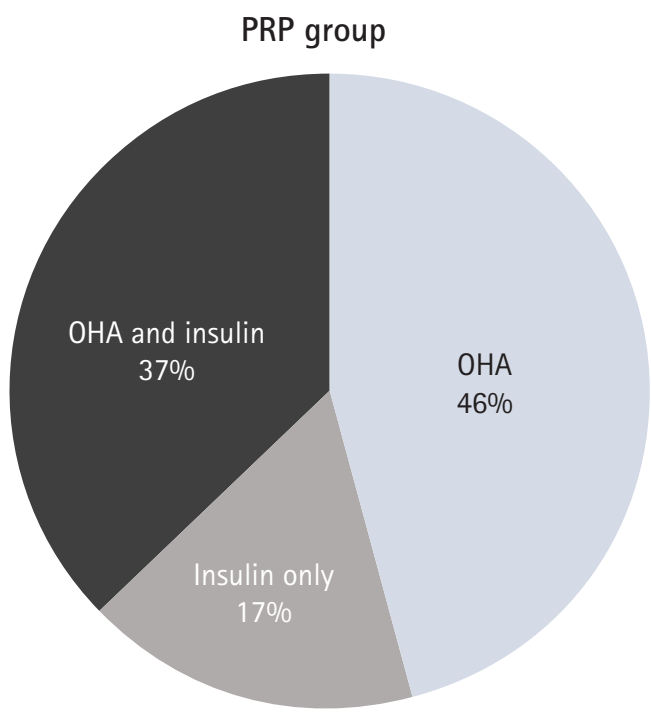

Fig. 2. Diabetic medications in people with adhesive capsulitis who received intra-articular platelet-rich plasma (PRP) injections. OHA: oral hypoglycaemic agents.

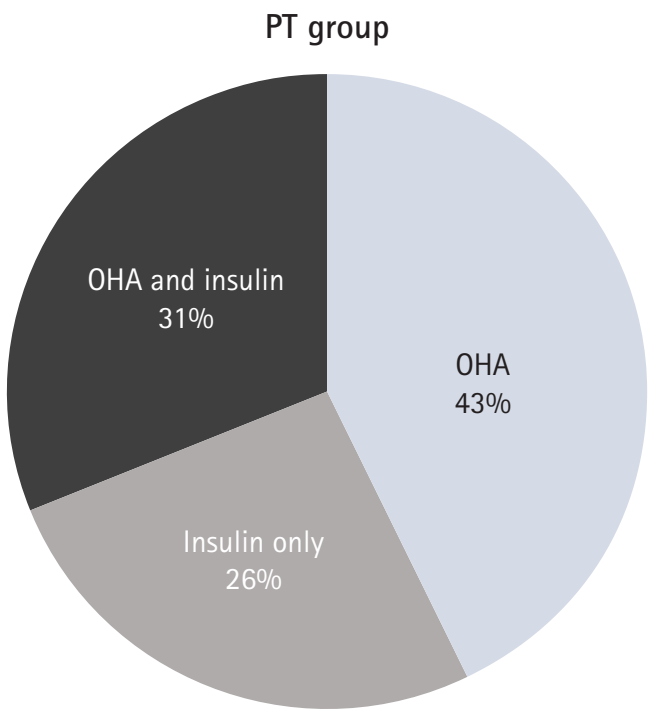

Fig. 3. Diabetic medications in people with adhesive capsulitis who received institution-based physical therapy (PT). OHA: oral hypoglycaemic agents.

Table 2. Study outcome data for the PRP and PT groups

\begin{tabular}{|c|c|c|c|c|c|c|c|}
\hline \multirow{2}{*}{ Variable } & \multicolumn{5}{|c|}{ PRP group $(n=35)^{*}$} & \multicolumn{2}{|c|}{ PT Group $(n=35)^{*}$} \\
\hline & Baseline visit & 2nd visit & 3rd visit & 4th visit & p-value $(\mathrm{RMA})^{\dagger}$ & Baseline visit & 2nd visit \\
\hline VAS & $73.1 \pm 8.5$ & $39.3 \pm 11.2$ & $24.2 \pm 8.7$ & $15.9 \pm 7.5$ & $<0.001$ & $74.4 \pm 7.5$ & $52.1 \pm 11.3$ \\
\hline \multicolumn{8}{|l|}{ Active $\operatorname{ROM}\left({ }^{\circ}\right)$} \\
\hline Flexion & $80.6 \pm 12.0$ & $98.6 \pm 11.8$ & $115.6 \pm 11.5$ & $129.9 \pm 11.5$ & $<0.001$ & $78.4 \pm 11.7$ & $88.9 \pm 14.3$ \\
\hline Extension & $22.3 \pm 6.2$ & $29.5 \pm 5.9$ & $35.5 \pm 5.8$ & $39.2 \pm 6.3$ & $<0.001$ & $21.4 \pm 2.9$ & $26.6 \pm 3.4$ \\
\hline Abduction & $68.0 \pm 8.3$ & $86.5 \pm 10.7$ & $101.7 \pm 12.4$ & $119.2 \pm 10.3$ & $<0.001$ & $64.8 \pm 9.1$ & $73.8 \pm 11.4$ \\
\hline Internal rotation & $16.8 \pm 6.2$ & $27.1 \pm 6.5$ & $37.1 \pm 7.7$ & $48.1 \pm 7.5$ & $<0.001$ & $18.0 \pm 5.3$ & $24.1 \pm 6.4$ \\
\hline External rotation & $17.8 \pm 3.8$ & $28.2 \pm 5.0$ & $38.8 \pm 8.1$ & $50.3 \pm 8.9$ & $<0.001$ & $19.1 \pm 4.5$ & $26.4 \pm 5.3$ \\
\hline \multicolumn{8}{|l|}{ Passive $\mathrm{ROM}\left({ }^{\circ}\right)$} \\
\hline Flexion & $97.9 \pm 13.5$ & $116.5 \pm 13.5$ & $137.0 \pm 12.5$ & $148.0 \pm 10.7$ & $<0.001$ & $95.0 \pm 10.6$ & $106.3 \pm 12.3$ \\
\hline Extension & $29.0 \pm 4.5$ & $37.9 \pm 6.6$ & $43.1 \pm 5.1$ & $45.8 \pm 3.7$ & $<0.001$ & $28.4 \pm 3.7$ & $33.1 \pm 4.7$ \\
\hline Abduction & $88.2 \pm 10.9$ & $104.2 \pm 11.0$ & $123.1 \pm 10.4$ & $138.3 \pm 9.4$ & $<0.001$ & $86.1 \pm 9.3$ & $96.6 \pm 11.7$ \\
\hline Internal rotation & $25.4 \pm 5.4$ & $39.3 \pm 6.1$ & $52.7 \pm 8.1$ & $62.1 \pm 9.0$ & $<0.001$ & $26.6 \pm 5.4$ & $34.6 \pm 5.9$ \\
\hline External rotation & $25.0 \pm 6.2$ & $39.1 \pm 7.7$ & $53.1 \pm 9.2$ & $61.7 \pm 10.1$ & $<0.001$ & $26.2 \pm 4.6$ & $34.5 \pm 6.0$ \\
\hline \multicolumn{8}{|l|}{ SPADI } \\
\hline Pain & $81.3 \pm 11.2$ & $47.1 \pm 9.6$ & $28.6 \pm 7.6$ & $17.0 \pm 7.2$ & $<0.001$ & $78.8 \pm 9.6$ & $65.2 \pm 10.7$ \\
\hline Disability & $64.3 \pm 13.4$ & $37.4 \pm 7.2$ & $25.2 \pm 8.0$ & $14.2 \pm 5.2$ & $<0.001$ & $63.1 \pm 11.2$ & $51.5 \pm 8.5$ \\
\hline Total & $71.1 \pm 122.0$ & $41.4 \pm 7.3$ & $26.4 \pm 6.4$ & $14.9 \pm 5.2$ & $<0.001$ & $69.3 \pm 10.3$ & $56.8 \pm 8.8$ \\
\hline
\end{tabular}

Values are presented as mean \pm standard deviation.

PRP: platelet-rich plasma, PT: physical therapy, RMA: repeated measures analysis, VAS: visual analog scale, ROM: range of motion, SPADI: shoulder pain and disability index.

${ }^{*}$ Intention-to-treat analysis (35 patients in each group); ${ }^{\dagger} \mathrm{RMA}$ of variance.

1 st to 4 th visits were statistically significant (Table 3). The improvements in mean ROM from 1 st to 2 nd, 1 st to $3 \mathrm{rd}$, and 1 st to 4 th assessments were greater in the PRP group (Table 3). At the conclusion of the study, there were significant differences $(\mathrm{p}<0.001)$ in ROM between the two groups (Table 4). A significant decrease in SPADI scores was seen (Tables 2 and 3 ) in the
PRP and PT groups. However, inter-group comparison revealed that the improvement was greater in the PRP group at the 4 th visit (Table 4).

During the study period, 17 patients (51.5\%) in the PRP group and six patients (18.7\%) in the PT group did not receive any acetaminophen tablets. During the entire study period, 11 patients 


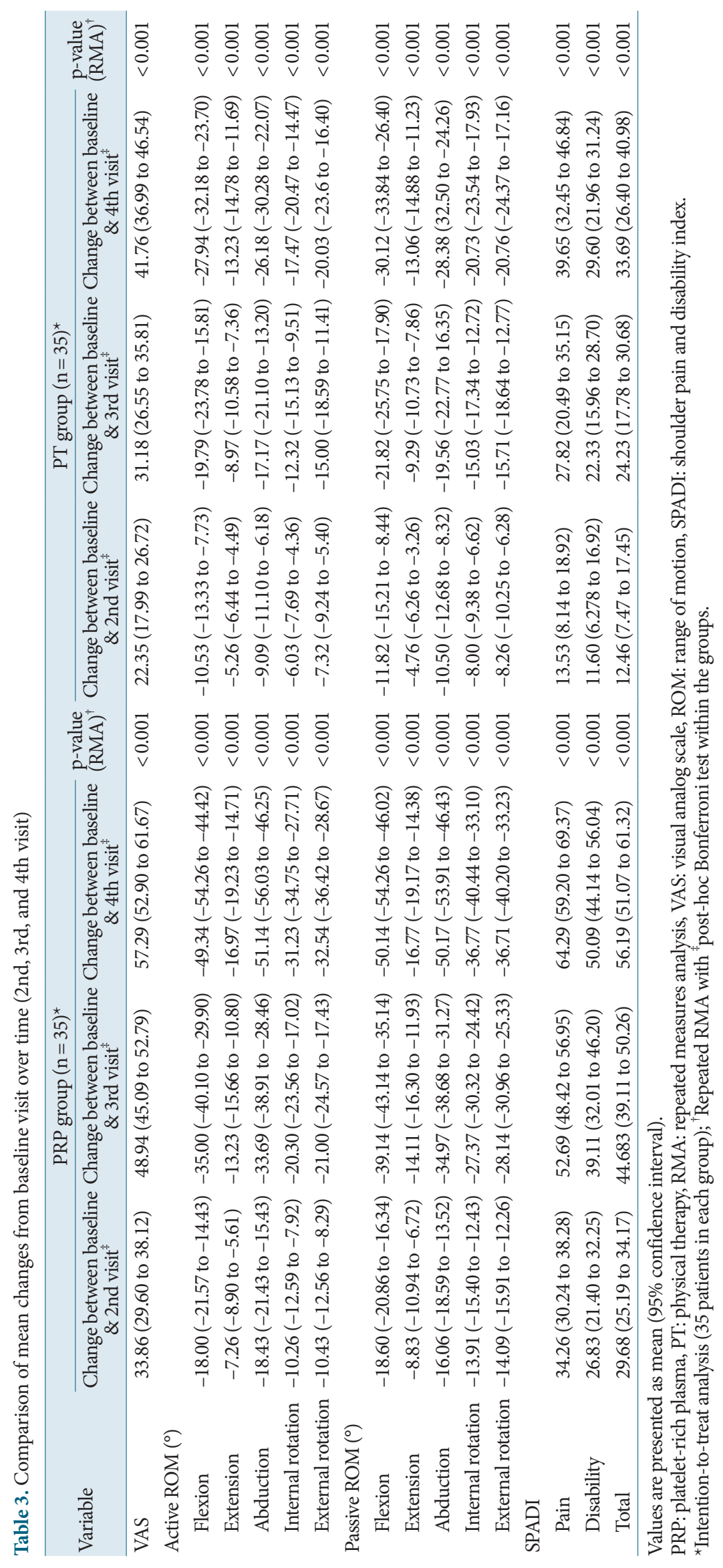


Table 4. Comparison of changes in outcome assessment scores (from baseline visit to 4th visit) at 12 weeks, between the two groups

\begin{tabular}{|c|c|c|c|c|}
\hline Variable & Change in PRP group $(n=35)$ & Change in PT group $(n=35)$ & Difference in change between groups & $\mathrm{p}$-value \\
\hline VAS & $57.28 \pm 9.26$ & $41.76 \pm 9.91$ & $-15.52(-20.13$ to -10.91$)$ & $<0.001$ \\
\hline \multicolumn{5}{|l|}{ Active $\mathrm{ROM}\left({ }^{\circ}\right)$} \\
\hline Flexion & $-49.34 \pm 10.38$ & $-27.94 \pm 8.80$ & $21.40(16.77$ to 26.03$)$ & $<0.001$ \\
\hline Extension & $-16.97 \pm 4.78$ & $-13.24 \pm 3.21$ & $3.73(1.77$ to 5.69$)$ & $<0.001$ \\
\hline Abduction & $-51.14 \pm 10.32$ & $-26.17 \pm 8.53$ & 24.97 (20.41 to 29.51$)$ & $<0.001$ \\
\hline Internal rotation & $-31.22 \pm 7.42$ & $-17.47 \pm 6.22$ & $13.76(10.46$ to 17.05$)$ & $<0.001$ \\
\hline External rotation & $-32.45 \pm 8.18$ & $-20.03 \pm 7.52$ & $12.51(8.73$ to 16.29$)$ & $<0.001$ \\
\hline \multicolumn{5}{|l|}{ Passive $\operatorname{ROM}\left({ }^{\circ}\right)$} \\
\hline Flexion & $-50.14 \pm 8.70$ & $-30.12 \pm 7.72$ & $20.02(16.07$ to 23.98$)$ & $<0.001$ \\
\hline Extension & $-16.77 \pm 5.06$ & $-13.06 \pm 3.79$ & $3.71(1.56$ to 5.86$)$ & $<0.001$ \\
\hline Abduction & $-50.17 \pm 7.90$ & $-28.38 \pm 8.55$ & $21.79(17.83$ to 25.74$)$ & $<0.001$ \\
\hline Internal rotation & $-36.77 \pm 7.75$ & $-20.74 \pm 5.82$ & 16.04 (12.73 to 19.34$)$ & $<0.001$ \\
\hline External rotation & $-36.71 \pm 7.35$ & $-20.76 \pm 7.49$ & 15.95 (12.38 to 19.52$)$ & $<0.001$ \\
\hline \multicolumn{5}{|l|}{ SPADI } \\
\hline Pain & $64.28 \pm 10.73$ & $39.64 \pm 14.94$ & $-24.64(-30.88$ to -18.40$)$ & $<0.001$ \\
\hline Disability & $50.09 \pm 12.56$ & $29.60 \pm 15.87$ & $-20.49(-27.36$ to -13.62$)$ & $<0.001$ \\
\hline total & $56.19 \pm 10.82$ & $33.69 \pm 15.14$ & $-22.50(-28.82$ to -16.19$)$ & $<0.001$ \\
\hline
\end{tabular}

Values are presented as mean \pm standard deviation or mean ( $95 \%$ confidence interval). The p-values correspond to the mean difference between two groups.

PRP: platelet-rich plasma, PT: physical therapy, VAS: visual analog scale, ROM: range of motion, SPADI: shoulder pain and disability index.

in the PRP group (33.3\%) received 1-2-g acetaminophen tablets and five PRP patients (15.1\%) received 3-4-g tablets. Whereas, in the PT group, three patients (9.4\%) received 1-2-g acetaminophen tablets, 10 (31.2\%) received 3-4-g tablets, and 13 (40.6\%) received $>4$-g tablets. Four patients in the PRP group reported mild pain and discomfort at the puncture site (immediately after injection). No significant complications (inflammation, infection, or other adverse events), either during the treatment or follow-up period, were reported in either group over the study period.

\section{DISCUSSION}

This study compared the effectiveness of a single PRP injection with an institution-based PT program for treating AC in diabetic patients. After receiving full treatment, both groups showed significant pain relief and functional improvement. However, compared with the institution-based PT programs, patients who received PRP injection showed rapid pain reduction, increased active and passive ROM, and improved shoulder function at all follow-up visits. At 12 weeks, the PRP group demonstrated clinically significant improvement in all parameters (pain relief, ROM, and shoulder function). Additionally, patients who received PRP injections consumed fewer acetaminophen tablets for pain relief than patients who received a PT program. Finally, none of the patients reported any significant complications after the intervention.

In terms of PRP injection efficacy for AC, this study's results were consistent with the majority of previously published studies [15-18,22,23]. However, most previous studies were conducted among patients with rotator cuff tendinopathy [17,22,23], where, in most cases, injections were given extra-articularly in the sub-acromial bursa [17,22,23]. Supra-scapular nerve block [24], sub-acromial bursa or intra-articular CS [2], or hyaluronic acid [3] injections have been studied extensively for shoulder AC in the general population. However, PRP injection efficacy for AC, especially in patients with DM, has not been demonstrated. To the best of our knowledge, this is the first study to confirm the beneficial effect of intra-articular PRP injection over institution-based PT programs for treatment of AC among diabetic patients.

It is already well established that PRP injection has anti-inflammatory, anti-nociceptive, and regenerative properties [15, 22,25]. Hepatocyte growth factor and tumor necrosis factor- $\alpha$, released from $\alpha$-granules in platelets, have potent anti-inflammatory properties [15,22]. Additionally, chemokines, released from platelets, regulate leucocyte recruitment at the inflammation site, which ultimately helps to reduce inflammatory and nociceptive reactions $[15,20,22,25]$.

On the other hand, few physical modalities have been reported 
as having pain relieving properties $[16,26,27]$. Though there is no clear consensus in the literature, UST and TENS are used in increased numbers for pain relief, and both have shown effectiveness [24]. PT sessions consisting of UST and TENS have been used to treat AC [16,24]. Exercise programs, including ROM and stretching exercises, are used to prevent further restriction and increase the ROM of the affected joint [28].

Strength training of the prime movers (shoulder joints) may prevent weakness and atrophy of the shoulder girdle muscles. Therefore, home self-guided exercise therapy, consisting of Codman exercises, stretching, and isometric strengthening exercise, was suggested to all study participants.

$\mathrm{AC}$ pathophysiology remains largely unknown, but most researchers believe that inflammation (followed by fibrosis of the joint capsule) is mainly responsible for AC $[15,16]$. Patients with $\mathrm{DM}$ report a higher incidence of $\mathrm{AC}$, probably due to poor circulation to the shoulder joint, abnormal collagen repair, and degenerative changes following tissue injury [4]. In this study, early treatment with intra-articular injection of PRP into the affected shoulder joint most probably reduced synovial proliferation, restricted capsular fibrosis, and altered the natural history of the disease. PRP is a reservoir of growth factors [12,15,22], including transforming growth factor- $\beta$, platelet-derived growth factor, a platelet-derived angiogenic factor, vascular endothelial growth factor, and fibroblast growth factor. These growth factors play a central role in tissue repair. However, additional clinical and basic science research is needed before recommending and/or framing clinical guidelines for PRP application in AC.

PRP quality was determined by the concentration of platelets in the PRP solution [15,22]. A four-fold increase in PRP platelets was achieved in this study, which is considered standard and adequate for PRP solutions for musculoskeletal intervention $[11,15,22]$. There is strong evidence that the higher the platelet count in the PRP, the greater the clinical response [29]. Blajchman [30] reported that storing platelets in freezing conditions might decrease their functional properties. In this study, PRP injection was administered within 30 minutes of its preparation. To date, there are no definite guidelines for using platelet-activating agents for intra-articular PRP injection.

The strengths of this study are that it focuses on diabetic patients, who are the most vulnerable to developing shoulder AC, and these patients usually refuse CS injection (the most common treatment). All PRP injections were administered under ultrasound guidance. ITT analysis was done, and outcomes were assessed at frequent intervals.

This study also has several limitations. Randomization was not done for participant recruitment. Participants decided for them- selves what treatment intervention they wanted (PRP injection vs. PT). The study was limited to a very short duration, 12 weeks, and an adequate control group was lacking, thus, the possibility of a placebo effect among the PRP injection patients cannot be excluded. A cost-benefit analysis of treatments was conducted but growth factor levels were not assessed in the prepared PRP solution. Randomized multicenter controlled trials with a longer follow-up duration are needed to confirm these results and reassess improvements from PRP injection, especially among diabetic patients.

In a diabetic population, PRP injection significantly improved shoulder pain and function compared with the institution-based PT program for shoulder AC. Furthermore, it is a safe and well-tolerated method for AC management in diabetic populations.

\section{ORCID}

Apurba Barman

Somnath Mukherjee

Mithilesh K Sinha

Jagannatha Sahoo

Amrutha Viswanath https://orcid.org/0000-0002-8990-1731

https://orcid.org/0000-0002-2272-5569

https://orcid.org/0000-0003-1803-5749

https://orcid.org/0000-0002-0489-895X

https://orcid.org/0000-0002-4765-7806

\section{REFERENCES}

1. Koh KH. Corticosteroid injection for adhesive capsulitis in primary care: a systematic review of randomised clinical trials Singapore Med J 2016;57:646-57.

2. Blanchard V, Barr S, Cerisola FL. The effectiveness of corticosteroid injections compared with physiotherapeutic interventions for adhesive capsulitis: a systematic review. Physiotherapy 2010;96:95-107.

3. Hsieh LF, Hsu WC, Lin YJ, Chang HL, Chen CC, Huang V. Addition of intra-articular hyaluronate injection to physical therapy program produces no extra benefits in patients with adhesive capsulitis of the shoulder: a randomized controlled trial. Arch Phys Med Rehabil 2012;93:957-64.

4. Cole A, Gill TK, Shanahan EM, Phillips P, Taylor AW, Hill CL. Is diabetes associated with shoulder pain or stiffness? Results from a population based study. J Rheumatol 2009;36:371-7.

5. Chan HB, Pua PY, How CH. Physical therapy in the management of frozen shoulder. Singapore Med J 2017;58:685-9.

6. Harris JD, Griesser MJ, Copelan A, Jones GL. Treatment of adhesive capsulitis with intra-articular hyaluronate: a systematic review. Int J Shoulder Surg 2011;5:31-7.

7. Lee DH, Yoon SH, Lee MY, Kwack KS, Rah UW. Capsule-preserving hydrodilatation with corticosteroid versus corticoste- 
roid injection alone in refractory adhesive capsulitis of shoulder: a randomized controlled trial. Arch Phys Med Rehabil 2017;98:815-21.

8. Waterbrook AL, Balcik BJ, Goshinska AJ. Blood glucose levels after local musculoskeletal steroid injections in patients with diabetes mellitus: a clinical review. Sports Health 2017;9:372-4.

9. Fitzpatrick J, Bulsara MK, McCrory PR, Richardson MD, Zheng $\mathrm{MH}$. Analysis of platelet-rich plasma extraction: variations in platelet and blood components between 4 common commercial kits. Orthop J Sports Med 2017;5:2325967116675272.

10. Filardo G, Kon E, Di Matteo B, et al. Platelet-rich plasma injections for the treatment of refractory Achilles tendinopathy: results at 4 years. Blood Transfus 2014;12:533-40.

11. Foster TE, Puskas BL, Mandelbaum BR, Gerhardt MB, Rodeo SA. Platelet-rich plasma: from basic science to clinical applications. Am J Sports Med 2009;37:2259-72.

12. Gautam VK, Verma S, Batra S, Bhatnagar N, Arora S. Platelet-rich plasma versus corticosteroid injection for recalcitrant lateral epicondylitis: clinical and ultrasonographic evaluation. J Orthop Surg (Hong Kong) 2015;23:1-5.

13. Dai WL, Zhou AG, Zhang H, Zhang J. Efficacy of platelet-rich plasma in the treatment of knee osteoarthritis: a meta-analysis of randomized controlled trials. Arthroscopy 2017;33:659-70.

14. Grambart ST. Sports medicine and platelet-rich plasma: nonsurgical therapy. Clin Podiatr Med Surg 2015;32:99-107.

15. Barman A, Mukherjee S, Sahoo J, et al. Single intra-articular platelet-rich plasma versus corticosteroid injections in the treatment of adhesive capsulitis of the shoulder: a cohort study. Am J Phys Med Rehabil 2019;98:549-57.

16. Kothari SY, Srikumar V, Singh N. Comparative efficacy of platelet rich plasma injection, corticosteroid injection and ultrasonic therapy in the treatment of periarthritis shoulder. J Clin Diagn Res 2017;11:RC15-8.

17. Scarpone M, Rabago D, Snell E, et al. Effectiveness of platelet-rich plasma injection for rotator cuff tendinopathy: a prospective open-label study. Glob Adv Health Med 2013;2:26-31.

18. Tahririan MA, Moezi M, Motififard M, Nemati M, Nemati A. Ultrasound guided platelet-rich plasma injection for the treatment of rotator cuff tendinopathy. Adv Biomed Res 2016;5:200.

19. Kasper DL, Fauci A, Hauser SL, Longo DL, Jameson JL, Lo- scalzo J. Harrison's principles of internal medicine. 19th ed. New York, NY: McGraw Hill Education; 2015.

20. Aslani MA, Mirzaee F, Baradaran AF, Zafarani Z, Aslani H. Clinical results of platelet-rich plasma in frozen shoulder. J Cell Mol Anesth 2020;5:74-8.

21. McCoy CE. Understanding the intention-to-treat principle in randomized controlled trials. West J Emerg Med 2017;18:10758.

22. Kesikburun S, Tan AK, Yilmaz B, Yaşar E, Yazicioğlu K. Platelet-rich plasma injections in the treatment of chronic rotator cuff tendinopathy: a randomized controlled trial with 1-year follow-up. Am J Sports Med 2013;41:2609-16.

23. Barreto RB, Azevedo AR, Gois MC, Freire MR, Silva DS, Cardoso JC. Platelet-rich plasma and corticosteroid in the treatment of rotator cuff impingement syndrome: randomized clinical trial. Rev Bras Ortop (Sao Paulo) 2019;54:636-43.

24. Klç Z, Filiz MB, Çakr T, Toraman NF. Addition of suprascapular nerve block to a physical therapy program produces an extra benefit to adhesive capsulitis: a randomized controlled trial. Am J Phys Med Rehabil 2015;94(10 Suppl 1):912-20.

25. Aslani H, Nourbakhsh ST, Zafarani Z, et al. Platelet-rich plasma for frozen shoulder: a case report. Arch Bone Jt Surg 2016;4:903.

26. Pajareya K, Chadchavalpanichaya N, Painmanakit S, Kaidwan C, Puttaruksa P, Wongsaranuchit Y. Effectiveness of physical therapy for patients with adhesive capsulitis: a randomized controlled trial. J Med Assoc Thai 2004;87:473-80.

27. Ryans I, Montgomery A, Galway R, Kernohan WG, McKane R. A randomized controlled trial of intra-articular triamcinolone and/or physiotherapy in shoulder capsulitis. Rheumatology (Oxford) 2005;44:529-35.

28. Le HV, Lee SJ, Nazarian A, Rodriguez EK. Adhesive capsulitis of the shoulder: review of pathophysiology and current clinical treatments. Shoulder Elbow 2017;9:75-84.

29. Arora S, Agnihotri N. Platelet derived biomaterials for therapeutic use: review of technical aspects. Indian J Hematol Blood Transfus 2017;33:159-67.

30. Blajchman MA. Novel platelet products, substitutes and alternatives. Transfus Clin Biol 2001;8:267-71. 